\title{
Imaging Diagnosis of Anomalous Total Coronary Artery From the Pulmonary Artery: Case Report
}

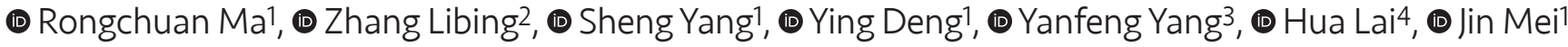 \\ 1University of Electronic Science and Technology of China, Faculty of Medicine; Chengdu Women's and Children's Central Hospital, Clinic of \\ Ultrasonic, Chengdu, China \\ 2University of Electronic Science and Technology of China, Faculty of Medicine; Chengdu Women's and Children's Central Hospital, Clinic of \\ Pediatric Surgical, Chengdu, China \\ 3University of Electronic Science and Technology of China, Faculty of Medicine; Chengdu Women's and Children's Central Hospital, Clinic of \\ Internal Medicine-cardiovascular, Chengdu, China \\ 4University of Electronic Science and Technology of China, Faculty of Medicine; Chengdu Women's and Children's Central Hospital, Clinic of \\ Radiology, Chengdu, China
}

\section{ABSTRACT}

Anomalous total coronary artery from the pulmonary artery is an extremely rare congenital coronary artery malformation. Only a few cases have been reported over the years, but with no comprehensive medical imaging data. We present the imaging findings of this case with transthoracic echocardiography, trans-esophageal echocardiography, computed tomography angiography (CTA) and cardiac catheterization.

Keywords: Anomalous total coronary artery from the pulmonary artery, echocardiography, CTA, cardiac catheterization

\section{Introduction}

Anomalous total coronary artery from the pulmonary artery (ATCAPA) is an extremely rare but lethal congenital coronary artery malformation (1,2). The patient's right coronary artery (RCA) and left coronary artery (LCA) have a common trunk that originated from the pulmonary artery. This could lead to myocardial ischemia and eventually death due to heart failure. Since the disease is rarely reported, the pathogenesis is unclear to date. This paper reports a detailed imaging diagnosis of ATCAPA as well as surgical treatment verification.

\section{Case Report}

A 6-month-old boy was hospitalized with symptoms of polypnea and cough. He had been treated at a local hospital 5 months previously for the neonatal pneumonia, and underwent trans-thoracic echocardiography (TTE) at the local hospital before being admitted to our clinic. Tertiary systolic murmur could be heard in the precardiac region on physical examination. The electrocardiogram showed right deviation of the electric axis, enl argement of the left ventricular and atrium, and abnormality of ST-segment (Figure 1). The BNP was up to $20,971.5 \mathrm{pg} / \mathrm{m}$. 
TTE showed spherical enlargement of the left ventricular, enlarged left atrium, and diffused incrassation of the endocardium ( $4.3 \mathrm{~mm}$ ). The mitral valve chordae tendineae and papillary muscle had become hyperechoic with severe mitral regurgitation and 40.3 percent-ejection fraction. The foramen oval was patent. There was no coronary artery arising from the right coronary sinus. A vessel originated from the right lateral wall of the main pulmonary artery trunk and tracked backward and downwards to the side of the left coronary sinus with no distinct boundary (Figure 2). It seemed the LCA and RCA arose from here. Three branches originated from proximal LCA. The RCA tracked between

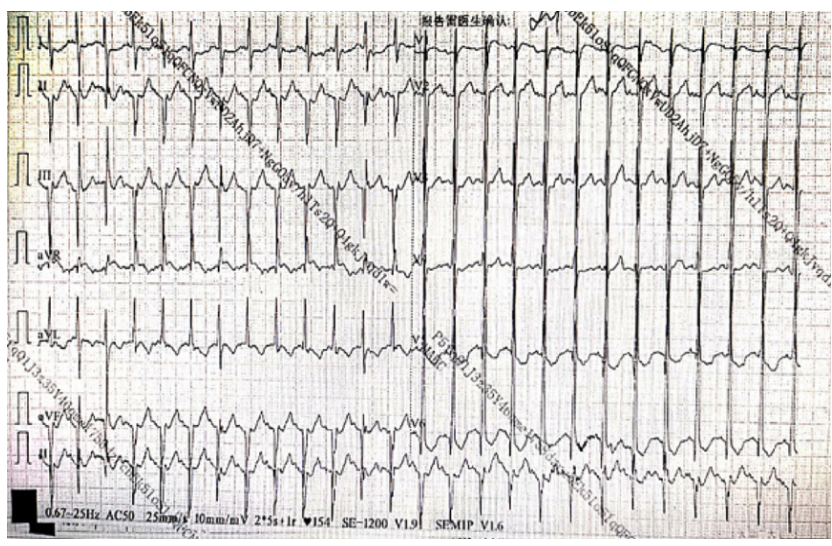

Figure 1. The preoperative ECG showing the right deviation of the electric axis, enlargement of the left ventricular and atrium, and abnormality of ST-segment ECG: Electrocardiogram

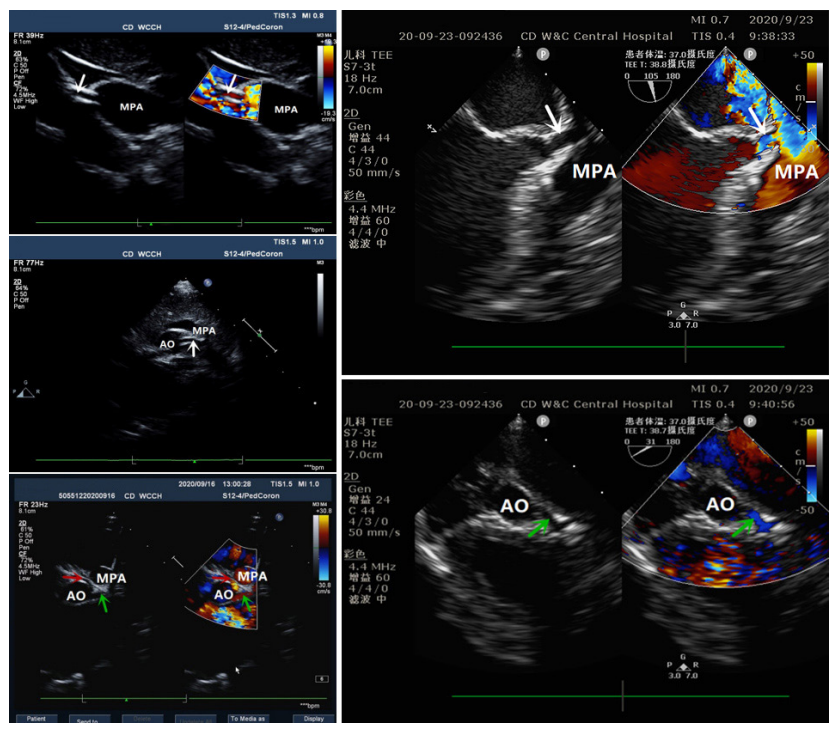

Figure 2. TTE and TEE showing a vessel (white arrow) originated from the right lateral wall of the main pulmonary artery (MPA) trunk, and tracked downwards close to the aorta $(A O)$, then branched LCA (green arrow) and RCA (red arrow)

TTE: Trans-thoracic echocardiography, TEE: Transesophageal echocardiography, RCA: Right coronary artery, LCA: Left coronary artery the aortic and pulmonary artery trunk. The direction of the coronary arteries flow was from proximal to distal. Intraoperative transesophageal echocardiography (TEE) revealed a vessel originating from the right posterior lateral wall and was tracked downwards to the side of the left coronary sinus with an intumescentia, then branched LCA and RCA (Figure 2). The direction of flow was shown to be consistent with the TTE.

The cardiac computed tomography angiography (CTA) showed the RCA and LCA with a common trunk originating from the pulmonary artery, and three branches of the LCA were observed (Figure 3). Cardiac catheterization was also performed for accurate diagnosis and to detect existing collateral circulation or not. Ascending aortography showed no coronary arteries originating from the aorta. The pulmonary arteriography revealed the same as CTA (Figure 3 ), and the length of the common trunk of the RCA and LCA was 3.2 millimeters. No definite collateral circulation was detected by the previously mentioned TTE, TEE, CTA, or cardiac catheterization.

The above mentioned imaging diagnoses were verified by surgical operation (Figure 4). The abnormality of the left ventricular endocardium and mitral apparatus coincided with the TTE findings. Nevertheless, the mural coronary arteries of both the proximal LCA and RCA had not been observed by the imaging examinations, but only by surgical operation. The common trunk of the RCA and LCA was holistically transferred to the aorta.

\section{Discussion}

ATCAPA, with no definite pathogenesis and incidence, is an extremely rare pathogeny of infantile myocardial ischemia. It is rarer than anomalous left coronary artery from the pulmonary artery (ALCAPA) with an incidence rate

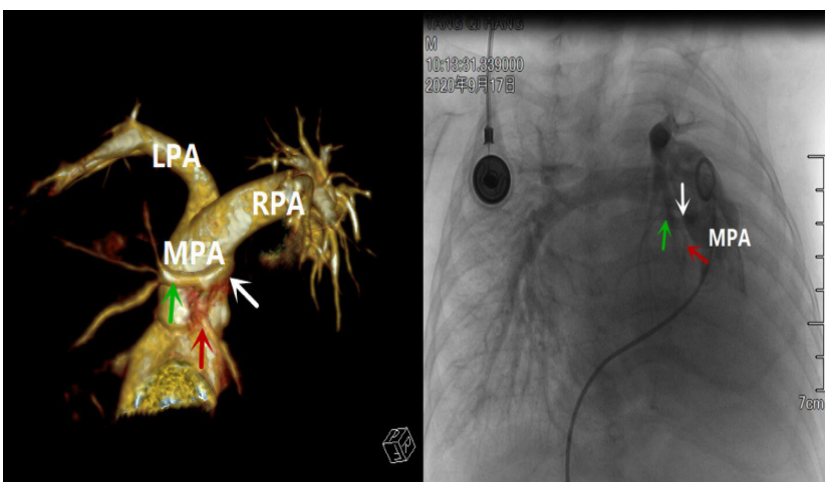

Figure 3. CTA and cardiac catheterization comforming the RCA (red arrow) and LCA (green arrow) with a common trunk (white arrow) originated from the main pulmonary artery (MPA)

CTA: Computed tomography angiography, RCA: Right coronary artery, LCA: Left coronary artery 


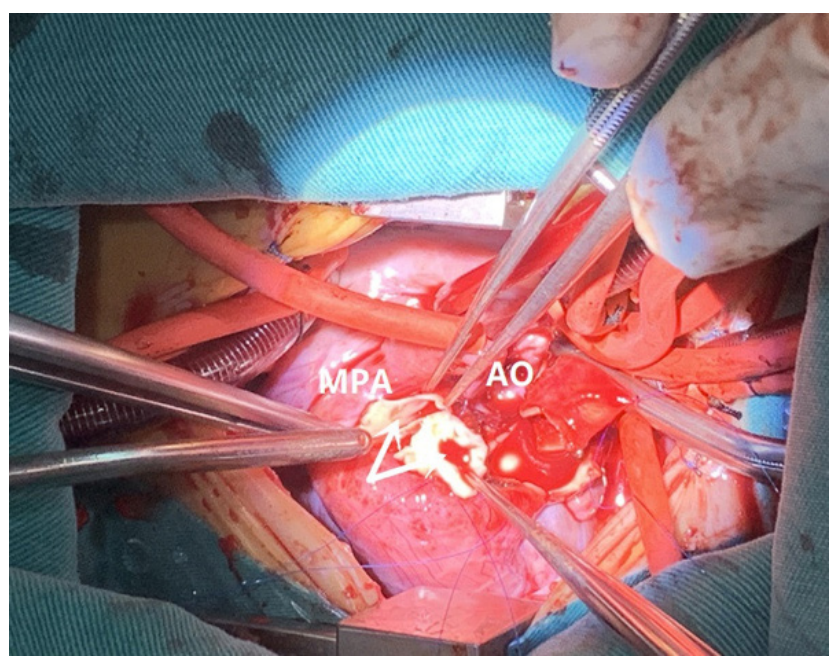

Figure 4. Tansfering the common trunk (white arrow) of RCA and LCA to the aorta by surgical operation

RCA: Right coronary artery, LCA: Left coronary artery

of $0.008 \%$ (3) and anomalous right coronary artery from the pulmonary artery (ARCAPA) with an incidence rate of $0.002 \%$ in the general population (2).

Those patients with ATCAPA have the co-pathogenic mechanism of ALCAPA and ARCAPA. The clinical symptoms of patients with ATCAPA are related to changes of relative pressure between systemic and pulmonary circulation and the level of coronary collateral circulation $(4,5)$. The main manifestations of the child in this case study are similar to the clinical symptoms in ALCAPA patients. Myocardial ischemia is progressively aggravated with increasing age (6). Incredibly, this patient survived to 6 months of age with hypoxic coronary flow from the pulmonary artery and no certain coronary collateral circulation. There are three possible explanations for this: (a) This patient has some coronary collateral circulations that could not be detected by imaging examinations and cardiac catheterization, (b) the long-term myocardial ischemia and left ventricular diastolic dysfunction with severe mitral regurgitation led to elevated left atrial filling pressure and, additionally, the patient, with repeated pulmonary infection, may have had increased pulmonary vascular resistance, which could enable the flow of pulmonary artery pump into the coronary artery during the diastolic period, (c) the patient's immature myocardium was able to better tolerate hypoxia. Due to long-term myocardial ischemia, the echocardiographic findings of this patient show spherical enlargement of the left ventricular and diffused incrassated endocardium. It should be carefully distinguished from endocardial fibroelastosis and dilated cardiomyopathy, which usually have normal coronary artery and no obvious incrassated endocardium in patients with dilated cardiomyopathy.

The gold standard for imaging diagnosis of anomalous origin of the coronary artery from the pulmonary artery is cardiac catheterization, which can clearly show the location of the origin of the coronary artery and judges the coronary flow direction. The CTA scan is comparable to cardiac catheterization except that it cannot indicate flow direction. Echocardiography is the first choice for imaging examinations for infant heart disease. In this case, both coronary arteries originated from the right back of pulmonary artery, and went close to left coronary sinus. Additionally, it was found in the operation that both proximal of the left and RCAs are partially mural coronary arteries. Such a situation could easily lead to the misdiagnosis that the coronary artery originates from the aortic sinus, which should be distinguished from our case carefully. The coronary artery should be carefully examined when an obvious growth of left ventricular and myocardial ischemia are detected by echocardiography in infants. CTA and cardiac catheterization should be integrated in the diagnostic process if necessary. The integration of multiple imaging techniques is of great value in the prompt detection and diagnosis of coronary artery origin diseases, the selection of operation time, as well as an improvement in prognosis.

\section{Ethics}

Informed Consent: The patient's parents approved to use this case for publication.

Peer-review: Externally peer-reviewed.

\section{Authorship Contributions}

Concept: J.M., Design: R.M., J.M., Data Collection or Processing: R.M., Z.L., S.Y., Y.D., Y.Y., H.L., M.J., Analysis or Interpretation: R.M., Writing: R.M.

Conflict of Interest: No conflict of interest was declared by the authors.

Financial Disclosure: The authors declared that this study received no financial support.

\section{References}

1. Heifetz SA, Robinowitz M, Mueller KH, Virmani R. Total anomalous origin of the coronary arteries from the pulmdonary artery. Pediatr Cardiol 1986; 7:11-8.

2. Xiao Y, Qiao W, Zhan Y, Zhang J, Ren W. Anomalous origin of both coronary arteries from the pulmonary artery in hypoplastic left heart syndrome. / Ultrasound Med 2015; 34:543-5.

3. Fan $H$, Jiakan W, Qicai $H$, Ximing Q, Huaidong C. Images of anomalous origin of the right coronary artery from the pulmonary artery. Heart Surg Forum 2019; 22:E308-9. 
4. Tavora F, Burke A, Kutys R, Li L, Virmani R. Total anomalous origin of the coronary circulation from the right pulmonary artery. Cardiovasc Pathol 2008; 17:246-9.

5. Januszewska K, Kehl HG, Malec E. Single ostium of the right and left coronary artery from the right pulmonary artery. Ann Thorac Surg 2018; 105:e67-9.
6. Yuan $\mathrm{XC}$, Hu J, Zeng $\mathrm{X}$, Zhou AY, Chen L. Echocardiographic diagnosis of anomalous origin of the left coronary artery from the pulmonary artery. Medicine (Baltimore) 2019; 98:e18046. 